Holzpelletanlagen: Moderne Ökotechnik mit traditionellem Brennstoff

\section{Dem Markt kräftig eingeheizt}

\section{Drei Prozent der Wärme in Deutschland wird aus Biomasse erzeugt. Dies ist das Zehnfache ihres Anteils an der Stromerzeugung. Dabei standen Holzheizungen und Kamine lange in dem Ruf, ineffizient und mit Gesundheits- und Geruchsbe- einträchtigungen verbunden zu sein. In den letzten Jahren wurden jedoch bei Holzpelletheizungen große Entwicklungsfortschritte gemacht.}

I Von Esther Hoffmann n Deutschland gibt es derzeit etwa 7 Millionen Kleinstfeuerungsanlagen wie Kaminöfen, Heizkamine oder Kachelöfen, die mit Holz betrieben werden können. Die Zahl der Holzzentralheizungen ist nicht bekannt. Die Holzfeuerungen werden zum überwiegenden Teil nur als Zweitoder Zusatzheizung eingesetzt. Dabei wird vor allem Brennholz und Waldrestholz genutzt, das nur zu geringen Anteilen über den Brennstoffhandel bezogen wird.

Holzfeuerungen, insbesondere Kleinanlagen, hatten jahrzehntelang ein negatives Image aufgrund von Nachbarschaftsbelästigung durch Rauch und Geruch. Die inzwischen erfolgte technische Weiterentwicklung von kleinen Holzheizanlagen wurde insbesondere in der Schweiz und in Österreich vorangetrieben. Wesentlich für die zunehmende Attraktivität war die Entwicklung von Holzpellets, die in Deutschland seit gut fünf Jahren als Brennstoff für Kleinstfeuerungsanlagen zugelassen sind. Holzpellets sind genormte zylindrische Presslinge, die in der Regel aus Holz- oder Sägespänen von holzverarbeitenden Betrieben hergestellt werden. Sie sind rieselfähig und eignen sich so zur Lieferung in Tankwagen und zur automatischen Befeuerung. Ihr Komfort ist mit öl- und gasbetriebenen Heizanlagen vergleichbar. Der geringe Wassergehalt und die Feinkörnigkeit ermöglichen eine optimale Verbrennung mit geringen Emissionen und Aschemengen. Gleichzeitig minimieren sie durch die automatische Beschickung Fehler durch unsachgemäße Bedienung oder Verbrennung von anderen Einsatzstoffen.

Die energetische Nutzung von Holzpellets ist nicht vollständig klimaneutral, da für Herstellung und Transport Energie aufgewendet werden muss. Bei Pellets aus trockenen Holzspänen oder Holzstaub liegt der Energieaufwand für die Herstellung bei etwa 3 Prozent ihres Energiegehalts.

Holzpelletheizanlagen können in Heizkessel und Raumheizer bzw. Primäröfen unterschieden wer- den. Pelletraumbeizer werden im Wohnbereich aufgestellt und erwärmen den Aufstellraum mittels Strahlungs- und Konvektionswärme. Aufgrund der niedrigen Wärmeleistung sind sie vor allem zur Beheizung von Wohnungen sowie Niedrigenergie- und Passivhäusern geeignet. Pelletheizkessel können als Zentralheizungsanlagen für Ein- und Mehrfamilienhäuser verwendet werden.

\section{Dynamischer Wachstumsmarkt ...}

Nach einer aktuellen Untersuchung des Instituts für ökologische Wirtschaftsforschung (1) wurden 2001 auf dem deutschen Markt etwa 7.000 Holzpelletfeuerungen abgesetzt, davon mehr als die Hälfte Raumheizer. Gegenüber den vorigen Jahren - 1999 knapp 1000, 2000 knapp 2000 Anlagen (2) - konnte der Absatz damit entscheidend gesteigert werden. Im Vergleich zur Gesamtzahl von jährlich etwa 400.000 verkauften Heizungen ist die Anzahl jedoch nach wie vor gering.

Die Investitionskosten von Holzpelletheizanlagen liegen zur Zeit deutlich über den Kosten für Gasoder Ölheizungen, demgegenüber sind die Brennstoffkosten für Pellets günstiger. Die günstigeren Brennstoffkosten führen allerdings erst nach mehr als 15 Jahren zu einer Amortisation der höheren Investitionskosten. Zentrale Einflussfaktoren für den rasanten Verkaufsanstieg waren zum einen die gestiegenen Ölpreise, zum anderen die Förderung von Pelletfeuerungen mit Zuschüssen aus dem Marktanreizprogramm zur Nutzung erneuerbarer Energien. Seit Juni letzten Jahres haben sich hier allerdings die Konditi-

Der deutsche Markt für Pelletheizungen ist zur Zeit noch stark - mit einem Anteil von mehr als 50 Prozent - durch österreichische Hersteller bestimmt. Deutsche Hersteller von Pelletheizungen waren bislang vor allem kleine Unternehmen, nun steigen auch große Heizungshersteller in den Markt ein. onen deutlich verschlechtert.

\section{... bald auch mit Blauem Engel}

Zwischen den verschiedenen Pelletheizungen gibt es große Unterschiede in Bezug auf Wirkungsgrade und Emissionen. Diese sind für VerbraucherInnen nicht leicht zu erkennen. Daher soll zukünftig das Umweltzeichen Blauer Engel für mehr Klarheit sorgen. Hierfür hat das IÖW im Auftrag des Umweltbundesamtes einen Vorschlag für Vergabekriterien entwickelt (1). Die Anforderungen umfassen Mindest- beziehungsweise Höchstwerte für Wirkungsgrade und Emissionen sowie den Stromverbrauch der Geräte. Darüber hinaus werden Anforderungen an die Bedienungsanleitung und begleitende Dienstleistungen vorgeschlagen. Die Vorschläge stießen bei Herstellern, Prüfinstituten und Verbänden auf reges Interesse, sodass mit der Verabschiedung der Vergabegrundlage noch in diesem Jahr gerechnet werden kann.

Ein Umweltzeichen für Holzpelletfeuerungen kann zum einen als Anreiz für technische Verbesserungen wirken, zum anderen stellt es für die Hersteller ein wichtiges Marketinginstrument dar. Dies ist insbesondere im Wettbewerb mit öl- und gasbefeuerten Heizungen bedeutsam, für die schon seit längerem Umweltzeichen vergeben werden.

Während bei der Stromerzeugung aus Biomasse, insbesondere in größeren Anlagen, die fehlende Wärmenachfrage oft zu einer nicht optimalen Ausnutzung des Brennstoffes führt, leisten Holzpelletheizanlagen durch den dezentralen Einsatz einen wichtigen Beitrag zum Klimaschutz, zur Ressourcenschonung und zum Ausbau der Nutzung erneuerbarer Energien.

\section{Anmerkungen}

(1) IÖW: Machbarkeitsstudie für neve Umweltzeichen für die Produktgruppe Holzpelletfeuerungen. Im Auftrag des Umweltbundesamtes. Bericht erscheint im Sommer 2002 als UBA-Texte Band

(2) CARMEN (Centrales Agrar-Rohstoff-Marketing- und Entwicklungs-Netzwerk): Holzpellets und Pelletheizanlagen 2000. Ergebnisse einer Umfrage. Straubing 2000.

\section{Die Autorin}

Esther Hoffmann ist wissenschaftliche Mitarbeiterin im Forschungsfeld Ökologische Unternehmenspolitik des Instituts für ökologische Wirtschaftsforschung.

Kontakt: IÖW, Potsdamer Str. 105, 10785 Berlin. Tel. 030/ 884594-22, Fax 030/ 8825439,

E-mail: esther.hoffmann@ioew.de 
(c) 20I0 Authors; licensee IÖW and oekom verlag. This is an article distributed under the terms of the Creative Commons Attribution Non-Commercial No Derivates License (http://creativecommons.org/licenses/by-nc-nd/3.o/), which permits unrestricted use, distribution, and reproduction in any medium, provided the original work is properly cited. 